\title{
Somatostatin Receptor Imaging of Neuroendocrine Tumors: From Agonists to Antagonists
}

\author{
Lisa Bodei ${ }^{1}$ and Wolfgang A. Weber ${ }^{2}$ \\ ${ }^{1}$ Molecular Imaging and Therapy Service, Department of Radiology, Memorial Sloan Kettering Cancer Center, New York, New York; \\ and ${ }^{2}$ Department of Nuclear Medicine, Technical University of Munich, Munich, Germany
}

See the associated articles on pages 909 and 915.

$\mathbf{T}$

he last 2 years have brought significant progress for the clinical use of somatostatin receptor (SSR) targeted imaging and therapy in the United States. In 2016, the Food and Drug Administration approved ${ }^{68} \mathrm{Ga}$-DOTATATE for imaging of neuroendocrine tumors (NETs), and very recently ${ }^{177} \mathrm{Lu}$-DOTATATE received approval for therapy of gastroenteropancreatic NETs. ${ }^{68} \mathrm{Ga} /{ }^{177} \mathrm{Lu}$-DOTATATE and the related peptides DOTATOC and DOTANOC are SSR ligands. They stimulate the receptor as the natural ligand somatostatin, which in turn causes internalization of the receptor-ligand complex (1). Internalization and intracellular retention were considered to be critical for highcontrast imaging of SSR-positive tumors because unbound peptide ligands are rapidly cleared via the kidneys, resulting in a very high gradient between activity concentration in the blood and activity concentration in the tumor tissue. Therefore, washout of cell surface-bound SSR ligands was believed to be fast and image contrast to be low or to be quickly decreasing over time.

However, G protein-coupled receptors, such as SSR, exist in different conformations, active and inactive, and receptor agonists and antagonists have different affinities for these conformations (2). Specifically, the affinity of the agonist is modulated by the degree of coupling of the receptor to the $G$ protein, whereas this is not the case for antagonists (2). These differences between $G$ protein-coupled receptor agonist and antagonist binding were demonstrated for opioid receptors in the 1970s (3), but only in 2006 did Ginj et al. demonstrate in cell culture studies that radiolabeled SSRs have severalfold more binding sites for antagonists than for agonists, probably because of the ability to recruit inactive receptors on the cell surface (1). Furthermore, SSR antagonists demonstrated significantly higher tumor uptake in mice than agonists did (1). A substantially higher number of binding sites was also shown by ex vivo autoradiography of human NET samples (4).

Received Feb. 19, 2018; revision accepted Mar. 21, 2018.

For correspondence or reprints contact: Wolfgang A. Weber, Department of Nuclear Medicine, Technical University of Munich, Ismaninger Strasse 22, 81675 Munich, Germany.

E-mail: ww_tum@outlook.com

Published online Mar. 30, 2018.

COPYRIGHT (C) 2018 by the Society of Nuclear Medicine and Molecular Imaging. DOI: 10.2967/jnumed.117.205161
This finding prompted the development and clinical testing of the high-affinity SSR type 2 (sstr2) ligand ${ }^{68}$ Ga-NODAGA-JR11 (NODAGA $=$ 1,4,7-triazacyclononane,1-glutaric acid-4,7-acetic acid and JR11 = Cpa-c(DCys-Aph(Hor)-DAph(Cbm)-Lys-ThrCys)-DTyr- $\mathrm{NH}_{2}$ ), which was tested clinically by Nicolas et al. $(5,6) .{ }^{68} \mathrm{Ga}-\mathrm{NODAGA}-J R 11$ had shown a highly favorable biodistribution in mice bearing sstr2-expressing xenografts and an almost 2 -fold-higher tumor uptake than the sstr 2 agonist ${ }^{68} \mathrm{Ga}$-DOTATATE, which has the same affinity for sstr2 as ${ }^{68} \mathrm{Ga}-N O D A G A-J R 11$ (7).

Small previous clinical studies suggested that SSR antagonists can efficiently target NETs and may be preferable to agonists. In a head-to-head comparison of the antagonist ${ }^{111}$ In-DOTA-BASS and ${ }^{111}$ In-DTPA-octreotide, the antagonist demonstrated improved image contrast and visualized significantly more metastatic lesions (25 vs. 17) than the agonist although both ligands exhibited almost identical SSR affinity (8). Tumor uptake of ${ }^{111}$ In-DOTA-BASS remained higher than that of ${ }^{111}$ In-octreotide until $24 \mathrm{~h}$ after injection (8), indicating that antagonists are well retained in the tumor tissue although they do not cause significant internalization of the SSR (7). In a comparison of JR11 labeled with ${ }^{177} \mathrm{Lu}\left({ }^{177} \mathrm{Lu}-\mathrm{DOTA}-\right.$ JR11) and ${ }^{177} \mathrm{Lu}$-DOTATATE in 4 patients with metastatic NETs, ${ }^{177} \mathrm{Lu}-\mathrm{DOTA}-J R 11$ delivered $1.7-10.6$ times higher tumor radiation doses per administered activity, again indicating that sstr2 antagonists can be retained by NETs for longer periods (9).

The 2 papers by Nicolas et al. in this issue of The Journal of Nuclear Medicine $(5,6)$ represent the first prospective, systematic evaluation of PET imaging with radiolabeled sstr2 antagonists. The first paper (5) describes the human biodistribution, safety, and radiation dosimetry of ${ }^{68} \mathrm{Ga}-\mathrm{NODAGA}-\mathrm{JR} 11$. Radiation doses to normal organs were similar to or lower than those in the literature for SSR agonists. Two masses of ${ }^{68} \mathrm{Ga}-N O D A G A-J R 11$ (15 and $50 \mu \mathrm{g}$ ) were injected in 12 patients, with no serious side effects for either peptide mass. However, it should be noted that a $50-\mu \mathrm{g}$ peptide mass is identical to a pharmacologically active dose of octreotide (which has an affinity for sstr2 similar to that of NODAGA-JR11). In patients with hormone-secreting tumors treated with octreotide, injection of ${ }^{68}$ Ga-NODAGA-JR11 may antagonize the inhibitory effects of octreotide on hormone secretion. Slow intravenous injection of ${ }^{68} \mathrm{Ga}-\mathrm{NODAGA}-J R 11$ may therefore be advisable in these patients until more safety data become available.

In the second paper, uptake in normal organs and tumor is compared between ${ }^{68}$ Ga-NODAGA-JR11 and the SSR agonist ${ }^{68} \mathrm{Ga}$-DOTATOC. The antagonist demonstrated significantly lower uptake than the agonist in liver, spleen, pancreas, and gastrointestinal tract. It is currently unclear whether this finding is due to the antagonist properties of ${ }^{68} \mathrm{Ga}-\mathrm{NODAGA}-\mathrm{JR} 11$ or to other differences in the pharmacologic properties of the two sstr2 ligands. 
Regardless, the low liver background activity is promising for detection of small liver metastases, which are notoriously difficult to delineate with agonists. Uptake of ${ }^{68} \mathrm{Ga}$-NODAGA-JR11 in several normal organs was slightly but significantly decreased by increasing the peptide mass from 15 to $50 \mu \mathrm{g}$. In contrast, tumor uptake of ${ }^{68} \mathrm{Ga}$-NODAGA-JR11 showed no significant change with increasing peptide mass. This result is consistent with previous animal studies on the impact of peptide mass on tumor and normal-tissue uptake of SSR ligands (10). A possible explanation is saturation of sstr2 receptors, ubiquitously expressed at low density in normal organs but not in the tumor. The stability of tumor uptake over a 3.3-fold range of injected masses is important for the clinical application of ${ }^{68} \mathrm{Ga}-\mathrm{NODAGA}-J R 11$ because the mass of NODAGA-JR11 injected for a given amount of radioactivity continuously increases with the physical decay of ${ }^{68} \mathrm{Ga}$. If tumor uptake of ${ }^{68} \mathrm{Ga}-\mathrm{NODAGA-JR} 11$ were highly dependent on the injected peptide mass, all quantitative indices of tracer uptake by the tumor tissue would be unstable.

Contrary to previous animal studies, tumor uptake of ${ }^{68} \mathrm{Ga}$ NODAGA-JR11 in patients was not higher than that of ${ }^{68} \mathrm{Ga}-$ DOTATATE (6), potentially indicating that tumor uptake is not limited by receptor density but by tracer delivery and perfusion. Nevertheless, markedly more liver metastases were detected by ${ }^{68} \mathrm{Ga}$-NODAGA-JR11 than by ${ }^{68} \mathrm{Ga}$-DOTATOC, because of the lower liver background activity (sensitivity, 94\% vs. 59\%). The positive predictive values of ${ }^{68} \mathrm{Ga}$-DOTATOC and ${ }^{68} \mathrm{Ga}$-NODAGAJR11 PET/CT were similarly high $(>95 \%)$, indicating that the additional number of lesions detected by ${ }^{68} \mathrm{Ga}-\mathrm{NODAGA-JR} 11$ were not false-positives.

These data are promising for NET staging with ${ }^{68} \mathrm{Ga}-\mathrm{NODAGA}-$ JR11 PET/CT. However, the absolute sensitivity reported for both ligands should be cautiously interpreted because the reference standard (CT and MRI) does not have a perfect sensitivity for detection of liver metastases (11). Another limitation of the study is the use of ${ }^{68} \mathrm{Ga}$-DOTATOC as a comparator for ${ }^{68} \mathrm{Ga}-\mathrm{NODAGA-JR} 11 .{ }^{68} \mathrm{Ga}$ DOTATOC also binds to sstr5 and to a lesser extent sstr3, whereas ${ }^{68}$ Ga-NODAGA-JR11 is a pure sstr2 ligand (7). Differences in biodistribution and tumor uptake may therefore reflect not only differences between their agonistic and antagonistic properties but also differences in the SSR binding profile.

Finally, the results of the present study support a potential theranostic role for radiolabeled SSR antagonists. The higher tumor uptake of JR11 than of DOTATOC suggests that higher tumor radiation doses may be delivered by antagonists, because there is also preliminary clinical evidence that antagonists show unexpectedly slow washout from NETs (9). However, larger studies are necessary to show that antagonists can provide meaningfully higher tumor response rates and prolonged progression-free survival. Also, the side-effect profile of radiolabeled antagonists needs to be studied carefully.

In conclusion, Nicolas et al. $(5,6)$ show that PET imaging of patients with SSR antagonists is promising for detection and staging of NETs. ${ }^{68} \mathrm{Ga}-\mathrm{NODAGA-JR} 11$ shows favorable biodistribution and may be superior to ${ }^{68} \mathrm{Ga}$-DOTATOC for detection of liver metastases. Further studies confirming the high sensitivity in larger populations, as well as studies on the impact of the higher sensitivity on patient management, are warranted.

\section{DISCLOSURE}

The authors acknowledge research support by Ipsen-Pharma, Paris, France. No other potential conflict of interest relevant to this article was reported.

\section{REFERENCES}

1. Ginj M, Zhang H, Waser B, et al. Radiolabeled somatostatin receptor antagonists are preferable to agonists for in vivo peptide receptor targeting of tumors. Proc Natl Acad Sci USA. 2006;103:16436-16441.

2. Perrin MH, Sutton SW, Cervini LA, Rivier JE, Vale WW. Comparison of an agonist, urocortin, and an antagonist, astressin, as radioligands for characterization of corticotropin-releasing factor receptors. J Pharmacol Exp Ther. 1999;288: 729-734.

3. Pasternak GW, Snyder SH. Identification of novel high affinity opiate receptor binding in rat brain. Nature. 1975;253:563-565.

4. Cescato R, Waser B, Fani M, Reubi JC. Evaluation of ${ }^{177}$ Lu-DOTA-sst2 antagonist versus ${ }^{177}$ Lu-DOTA-sst2 agonist binding in human cancers in vitro. J Nucl Med. 2011;52:1886-1890.

5. Nicolas GP, Beykan S, Bouterfa H, et al. Safety, biodistribution, and radiation dosimetry of ${ }^{68} \mathrm{Ga}$-OPS202 in patients with gastroenteropancreatic neuroendocrine tumors: a prospective phase I imaging study. J Nucl Med. 2018;59:909-914.

6. Nicolas GP, Schreiter N, Kaul F, et al. Sensitivity comparison of ${ }^{68} \mathrm{Ga}-\mathrm{OPS} 202$ and ${ }^{68} \mathrm{Ga}$-DOTATOC PET/CT in patients with gastroenteropancreatic neuroendocrine tumors: a prospective phase II imaging study. J Nucl Med. 2018;59:915-921.

7. Fani M, Braun F, Waser B, et al. Unexpected sensitivity of sst 2 antagonists to N-terminal radiometal modifications. J Nucl Med. 2012;53:1481-1489.

8. Wild D, Fani M, Behe M, et al. First clinical evidence that imaging with somatostatin receptor antagonists is feasible. J Nucl Med. 2011;52:1412-1417.

9. Wild D, Fani M, Fischer R, et al. Comparison of somatostatin receptor agonist and antagonist for peptide receptor radionuclide therapy: a pilot study. $\mathrm{J} \mathrm{Nucl}$ Med. 2014;55:1248-1252.

10. Nicolas GP, Mansi R, McDougall L, et al. Biodistribution, pharmacokinetics, and dosimetry of ${ }^{177} \mathrm{Lu}-,{ }^{90} \mathrm{Y}-$, and ${ }^{111} \mathrm{In}$-labeled somatostatin receptor antagonist OPS201 in comparison to the agonist ${ }^{177}$ Lu-DOTATATE: the mass effect. $J$ Nucl Med. 2017;58:1435-1441.

11. Elias D, Lefevre JH, Duvillard P, et al. Hepatic metastases from neuroendocrine tumors with a "thin slice" pathological examination: they are many more than you think. Ann Surg. 2010;251:307-310. 